\title{
Protein Supplementation and the Incidence of Apoptosis and Oxidative Stress in Mouse Embryos
}

\author{
Navid Esfandiari, DVM, PhD, Tommaso Falcone, MD, Ashok Agarwal, PhD, Marjan Attaran, MD, \\ David R. Nelson, MS, and Rakesh K. Sharma, $\mathrm{PhD}$
}

OBJECTIVE: To estimate the effect of protein supplementation of culture media on reactive oxygen species production and incidence of apoptosis in preimplantation mouse embryos.

METHODS: A total of 72 two-cell mouse embryos were cultured in human tubal fluid (HTF) alone (HTF-alone, control) and 71 embryos in HTF with protein supplementation (10\% serum substitute supplement; HTF-SSS) for 72 hours. Total cell number per embryo was determined by staining with Hoechst 33258. Allocation of inner cell mass and trophectoderm in blastocysts and incidence of apoptosis were determined by confocal microscopy. Levels of reactive oxygen species in culture media were measured by chemiluminescence assay using luminol as probe.

RESULTS: Blastocyst development, total cell number, and the inner cell mass/trophectoderm ratio were similar between the 2 groups. The blastocyst hatching rate was significantly higher in the HTF-SSS group than in the HTF-alone group $(20 \%$ compared with $4 \%, P=.007)$. Level of reactive oxygen species was significantly higher in HTF-alone compared with HTF-SSS at 24 hours (median and interquartile range $28[13,43]$ compared with $0[0,1], P=.02), 48$ hours (24 [21, 26] compared with $2[1,2], P=.02)$, and 72 hours $(26[9,32]$ compared with $2[2,3], P=.03)$. The incidence of apoptosis in blastocysts cultured in HTF-SSS was significantly lower than those in HTF-alone group (mean \pm standard deviation $2.38 \pm 0.68$ and $5.81 \pm 1.11$, respectively, $P=.001)$.

CONCLUSION: Protein supplementation of culture media improves the hatching rate and reduces reactive oxygen species levels and the incidence of apoptosis in mouse preimplantation embryos. (Obstet Gynecol 2005;105: 653-60. (C) 2005 by The American College of Obstetricians and Gynecologists.)

The quality of in vitro-produced blastocysts continually lags behind those of blastocysts produced in vivo. ${ }^{1,2}$

From the Department of Obstetrics and Gynecology, Glickman Urological Institute, and Department of Biostatistics and Epidemiology, The Cleveland Clinic Foundation, Cleveland, Ohio.

Presented at the 59th Annual Meeting of the American Society for Reproductive Medicine, October 12-17, 2002, Seattle, Washington.
Postfertilization culture environment has a huge impact on the blastocyst formation, irrespective of the origin of the zygote. $^{3}$ In vitro culture conditions, although capable of producing blastocysts in relatively high numbers, are far from optimal. ${ }^{4,5}$ These differences are manifested at the level of morphology, ${ }^{6}$ metabolism, ${ }^{7}$ and gene expression. ${ }^{8}$

One of the major differences between in vivo and in vitro environment for the embryo is oxygen tension. ${ }^{7}$ The relatively high oxygen concentrations in the microenvironment surrounding preimplantation embryos in vitro may disturb the balance between the formation of reactive oxygen species and antioxidants, leading to oxidative stress. ${ }^{9-11}$ Oxidative stress has been implicated in many different types of injuries, including membrane lipids peroxidation, oxidation of amino acids and nucleic acids, apoptosis, and necrosis. Cell death by apoptosis is a physiologic phenomenon occurring during several processes, including embryogenesis. Although apoptosis takes place as a normal part of embryonic development in vivo, but it is more likely to occur during in vitro embryo culture due to suboptimal conditions. ${ }^{12-14}$ Apoptosis is characterized by DNA fragmentation, cell shrinkage, and multiple cellular fragments, and more frequent apoptosis is seen in blastocysts cultured in vitro than in vivo. ${ }^{15}$

Normally, both oocytes and embryos are protected against oxidative stress by oxygen radical scavengers that are present in follicular and oviductal fluids. ${ }^{11} \mathrm{~A}$ large number of antioxidants have been studied in bovine and murine embryo culture such as $\beta$-mercaptoethanol, ${ }^{16}$ cysteamine,${ }^{17}$ vitamins $\mathrm{C}$ and $\mathrm{E},{ }^{18}$ taurine,${ }^{19}$ allopurinol, ${ }^{20}$ hypotaurine,${ }^{21}$ thiols, ${ }^{16,17,22,23}$ and superoxide dismutase. ${ }^{21,24,25}$ Despite the undefined and variable nature of serum composition, the supplementation of embryo culture media with serum is practiced widely. The complex molecules comprising serum can maintain embryo function by preventing cellular stress due to suboptimal culture conditions in vitro. Overexpression of superoxide dismutase protects embryos from cell death in different systems. ${ }^{26}$ 
The objective of our study was 1) to clarify the role of protein supplementation (serum substitute supplement) on preimplantation embryo development, ie, early, expanding, and expanded blastocysts using hatching as the endpoint and 2) to assess its effect on reactive oxygen species production and incidence of apoptosis in cultured embryos.

\section{MATERIALS AND METHODS}

Frozen 2-cell mouse embryos were obtained from B6C3F1 mouse crossed with B6D2F1 mouse. A culture system using 2-cell mouse embryos was used to evaluate the effects of protein supplementation in culture medium. Cryopreserved mouse embryos (Embryotech Laboratories, Inc., Wilmington, MA) were used. The straws were thawed, and embryos with intact blastomeres were used for the study. Embryos were incubated in 2 groups: human tubal fluid (HTF, Irvine Scientific, Santa Ana, CA) alone (HTF-alone) $(\mathrm{n}=72)$ and $\mathrm{HTF}$ with $10 \%$ serum substitute supplement, (HTFSSS) $(\mathrm{n}=71)$.

Culturing embryos in groups enhances their development potential. In the HTF-alone group, 9 sets consisting of 8 embryos $/ \mathrm{mL}$ of culture medium in each set totaling 72 embryos were used. In the HTF-SSS group also, 9 sets were used. Of these, 8 sets had 8 embryos each $(64$ embryos) and 1 set had 7 embryos, giving a total of 71 embryos. Before starting the culture, each dish containing $1 \mathrm{~mL}$ of culture medium was incubated overnight for equilibration at $37^{\circ} \mathrm{C}$ in $5 \% \mathrm{CO}_{2}$ in air (20\%). Embryos in both groups were monitored daily at the same time in the morning using Normarski and bright field inverted optics. The number of embryos cleaving to 4- to 8-cell, morula, blastocysts (early, expanding, expanded), and hatching as endpoint was recorded. We only observed the hatching process and did not study the embryos after hatching.

Levels of reactive oxygen species in culture media were measured after 0 hours (baseline), 24 hours, 48 hours, and 72 hours by the chemiluminescence assay method using luminol (5-amino-2,3-dihydro-1,4-phthalazinedione; Sigma Chemical Co., St. Louis, MO) as the probe. ${ }^{27}$ Ten microliters of $5 \mathrm{mM}$ luminol was prepared in dimethyl sulfoxide (Sigma Chemical Co.). Aliquots of $400 \mu \mathrm{l}$ of culture media were prepared in duplicate along with the blank and control. Blank consisted of $400 \mu \mathrm{l}$ of phosphate buffered saline (PBS, pH 7.4) instead of culture medium, and control consisted of an equal volume $(400 \mu \mathrm{l})$ of PBS and $10 \mu \mathrm{l}$ luminol. Luminol combines with hydrogen peroxide, superoxide anion, and the hydroxyl radicals present in the culture medium to produce the photons. These were measured in the integrated mode for 15 minutes using a luminometer (LKB 953, Wallac Inc., Gaithersburg, MD). The amount of reactive oxygen species produced was expressed as $10^{4}$ photons counted per minute (cpm). ${ }^{27}$

Individual embryos were stained with the terminal deoxynucleotide transferase (TdT)-mediated deoxyuridine triphosphate (dUTP)-biotin nick end-labeling (TUNEL) technique (in situ cell death detection system; Roche Diagnostic Corporation, Indianapolis, IN). To distinguish apoptosis from necrosis, embryos were stained with propidium iodide (Sigma Chemical Co.) before the TUNEL assay was performed. Embryos were incubated in $20 \mu \mathrm{g} / \mathrm{mL}$ propidium iodide in HTF for 10 minutes. Propidium iodide is membrane impermeant and generally excluded from viable cells. It is commonly used for identifying dead cells in a population and as a counterstain in multicolor fluorescence techniques. The plasma membrane integrity is lost in necrotic but not apoptotic cells. We validated apoptosis by exclusion of propidium iodide stain. ${ }^{28}$

After overnight fixation at $4^{\circ} \mathrm{C}(3.7 \%$ paraformaldehyde in PBS), embryos were washed thrice in PBS containing $0.3 \%$ polyvinylpyrrolidone and permeabilized in $0.5 \%$ Triton X-100 on ice for 2 minutes. After repeating the washing in PBS with polyvinylpyrrolidone, the embryos were incubated in TUNEL reaction cocktail at $37^{\circ} \mathrm{C}$ for 1 hour in the dark.

The total blastomere count per embryo was determined by staining the embryos with bisbenzimide (Hoechst dye 33258, Sigma Chemical Co.). The embryos were washed extensively and mounted with slight coverslip compression in Vectashield anti-bleaching solution (Vector Labs, Burlingame, CA). The slides were sealed with clear nail polish and stored at $4^{\circ} \mathrm{C}$ in the dark for up to 1 week until confocal analysis.

Images were collected with a Leica TCS-SP2 laser scanning spectral confocal microscope (Leica Lasertechnik GmbH, Heidelberg, Germany) using an HCX Plan Apo 63X, 1.32 NA objective at a zoom of 2. The specimen was excited at $364 \mathrm{~nm}$ (ultraviolet) for bisbenzimide, $488 \mathrm{~nm}$ for TUNEL-fluorescein isothiocyanate, and $514 \mathrm{~nm}$ for propidium iodide. The emitted fluorescence from each of the 3 probes was detected with 3 separate photomultiplier detectors, whose spectrophotometer slits were set for 400-490, 500-550, and 585-810 nm, respectively.

The whole blastocyst was captured as a $Z$ series of optical sections from the top of the blastocyst to the bottom. A projection of the 3-dimensional stack of images was then created with the Leica software. Images were collected sequentially at each level of the specimen to prevent cross 
Table 1. The Effect of Protein Supplementation on Mouse Embryo Development

\begin{tabular}{lcccc}
\hline Group & $\begin{array}{c}\text { Morula } \\
(48 \mathrm{~h})\end{array}$ & $\begin{array}{c}\text { Blastocyst } \\
(48 \mathrm{~h})\end{array}$ & $\begin{array}{c}\text { Blastocyst } \\
(72 \mathrm{~h})\end{array}$ & $\begin{array}{c}\text { Hatching Blastocyst } \\
(72 \mathrm{~h})\end{array}$ \\
\hline HTF-alone & $37(53)$ & $34(47)$ & $66(92)$ & $3(4)$ \\
HTF-SSS & $46(68)$ & $23(32)$ & $65(92)$ & $14(20)$ \\
$P$ & .02 & .13 & .9 & .007 \\
\hline
\end{tabular}

HTF, human tubal fluid; SSS, serum substitute supplement.

Values are $\mathrm{n}(\%)$.

$P<.05$ was considered significant using logistic regression with generalized estimating equations.

talk of the fluorophores. These were collected along the $\mathrm{Z}$ axis of the sample with a step size of 1-3 pm.

Each optical section of the blastocyst was analyzed for total number of nuclei and number of TUNEL-labeled nuclei in inner cell mass and trophectoderm. Trophectoderm nuclei were distinguished from inner cell mass nuclei by a combination of examination of their position and morphology. ${ }^{29}$ In general, trophectoderm nuclei are smaller and more intensely stained, whereas inner cell mass nuclei are larger, rounded, and less intensely stained.

Experiments were designed in a clustered manner in that 8 embryos were used in each trial clustered within the complete experiment. All statistical analyses used the fact that per-embryo findings were clustered with other embryos during each trial. Therefore, comparisons between treatments used treatment as the sole independent variable, with embryos treated as clustered observations within each experiment. Mixed model analysis of variance was performed for calculating total cell number (continuous dependent variable) and logistic regression using generalized estimating equation for calculating percentage blastocyst development rate (binary dependent variable). These analyses used each experiment as a clustered variable within each treatment to adjust for any within-trial correlation and treatment as the sole independent variable. A compound symmetry model was used to model the correlation structure. Summary statistics for these variables are presented as mean and standard deviation, with the exception of total cells. Total cells was not normally distributed and transformed with base-10 logarithms, and summary statistics are presented as median and interquartile range (IQR).
Correlations of reactive oxygen species with other variables were performed with Spearman's nonparametric method. Clustered data methodology was not used because reactive oxygen species was measured as the trial level (rather than per embryo) and correlated with the average of the measures of the 8 embryos. Reactive oxygen species comparisons were performed with Wilcoxon rank sum tests, and summary statistics for these variables are presented as median and IQR.

Statistical significance was assessed with 2-tailed tests, using $P<.05$ as Type I error rates. The sample size had $90 \%$ power to detect a difference of 1.5 apoptotic cells per blastocyst between groups, and 90\% power to detect a $0.17 \times 10^{4} \mathrm{cpm}$ difference in reactive oxygen species. Initial trial experiments indicated a between-trial standard deviation of 0.75 apoptotic cells per blastocyst, and $0.085 \times 10^{4}$ $\mathrm{cpm}$ between-trial standard deviation in reactive oxygen species. The sample size calculations were performed on a per trial basis to be conservative. Mixed and generalized estimating equation models should be more powerful than analyses on the level of trial that would ignore the withintrial clusters. Calculations were performed with SAS 8.1 software (SAS Institute Inc., Cary, NC).

\section{RESULTS}

The percentage and number of embryos cleaving to 4- to 8-cell, morula, blastocyst, and hatching blastocysts in HTF-alone and HTF-SSS media are shown in Table 1. The percentage of blastocyte formation in a given period of time ( 48 hours and 72 hours) was not affected by protein supplementation. The early blastocyst formation after 48 hours was higher (47\%) but not significant in the

Table 2. The Allocation of Inner Cell Mass and Trophectoderm in Blastocyst After Culture in HTF-alone and HTF-SSS

\begin{tabular}{lcccc}
\hline \multicolumn{1}{c}{ Group } & Total Cell Number & Inner Cell Mass & Trophectoderm & $\begin{array}{r}\text { Inner Cell Mass/ } \\
\text { Trophectoderm }\end{array}$ \\
\hline HTF-alone & $55.36 \pm 2.80$ & $14.37 \pm 0.75$ & $41.89 \pm 1.99$ & $0.35(0.03)$ \\
HTF-SSS & $52.57 \pm 8.68$ & $13.61 \pm 1.50$ & $39.18 \pm 2.81$ & $0.37(0.02)$ \\
$P$ & .36 & .16 & .046 & .91 \\
\hline
\end{tabular}

HTF, human tubal fluid; SSS, serum substitute supplement.

Values are mean \pm standard deviation and $\mathrm{n}(\%)$.

$P<.05$ was considered significant using mixed-model analysis of variance. 


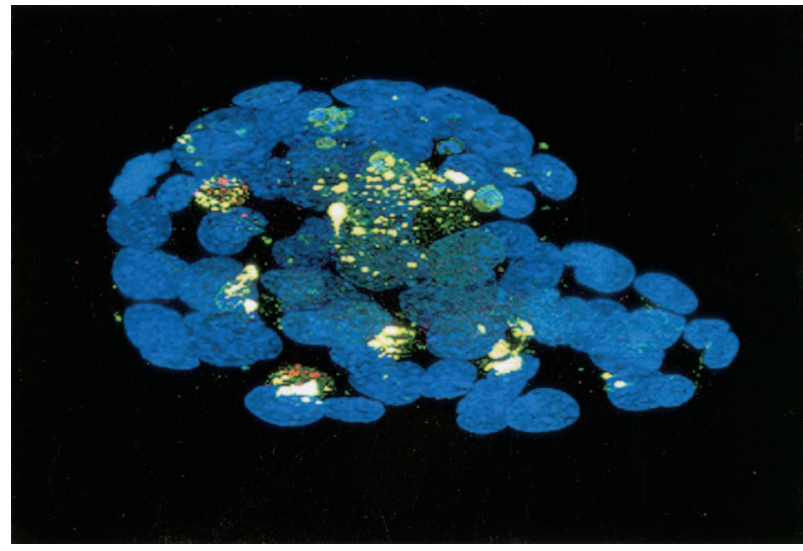

Fig. 1. Confocal photomicrograph of mouse hatching blastocyst stained for apoptosis ( $\times 400$, original magnification). The embryo is labeled with bisbenzimide, terminal deoxynucleotide transferase (TdT)-mediated deoxyuridine triphosphate (dUTP)-biotin nick end-labeling (fluorescein isothiocyanate), and propidium iodide. All nuclei of the blastocyst are labeled with bisbenzimide (b/ue channel) and apoptotic nuclei with terminal deoxynucleotide transferase (TdT)-mediated deoxyuridine triphosphate (dUTP)biotin nick end-labeling (green channel).

Esfandiari. Protein Supplementation in Embryos. Obstet Gynecol 2005.

embryos that were cultured in HTF-alone compared with those that were cultured in HTF-SSS (32\%). However, after 72 hours, the overall blastocyst development rate (binary dependent variable) was similar in the 2 culture systems. The hatching rate was significantly higher in HTF-SSS compared with the embryos that were cultured in HTF-alone $(P=.007)$. During the in vitro culture, no cytoplasmic fragmentation was observed in either group.

The total cell number for the embryos that were cultured in the 2 systems is shown in Table 2. The total cell number in the HTF-SSS group was similar to that of the blastocysts that were cultured in HTF-alone. However, the hatching blastocysts that were cultured in HTFSSS had a significantly higher total cell number (median and IQR $70.5[67,73])$ than embryos cultured in HTF- alone $(63[60,66], P=.04)$. The inner cell mass and inner cell mass/trophectoderm ratio in HTF-SSS group was similar to HTF-alone group (Table 2).

Baseline levels of reactive oxygen species at 0 hours were similar in the 2 culture media. However, significantly higher levels of reactive oxygen species $\left(10^{4} \mathrm{cpm}\right)$ were seen in HTF-alone compared with those in HTF-SSS at 24 hours (median and IQR $28[13,43]$ compared with $0[0,1], P=$ $.02), 48$ hours $(24[21,26]$ compared with $2[1,2], P=.02)$, and 72 hours $(26[9,32]$ compared with $2[2,3], P=.03)$.

Positive TUNEL labeling in apoptotic cells was defined as a bright fluorescence-staining pattern (Fig. 1). We did not exclude any of the blastocyst because of extensive propidium iodide staining. Positive labeling was observed in $98 \%$ of the blastocysts that were cultured in HTF-alone and in 94\% of the blastocysts that were cultured in the HTF-SSS media. However, in the growth-retarded or arrested embryos before morula stage, a positive labeling could not be identified. Apoptosis was first detected at the morula stage in the growthretarded embryos.

Protein supplementation reduced the incidence of apoptosis in the cultured embryos, as evidenced by the significantly lower percentage of apoptosis in the blastocysts (Table 3) and of apoptotic cells per blastocyst (mean and standard deviation $2.38 \pm 0.68$ in HTF-SSS and $5.81 \pm$ 1.11 in HTF-alone, $P=.001)$. Positive TUNEL labeling was seen in both the inner cell mass and trophectoderm in both groups. Cell death in the blastocysts occurred primarily in the inner cell mass in both groups.

No significant correlation was seen between the levels of reactive oxygen species and embryo development in the HTF-SSS group. There was a positive correlation between the levels of reactive oxygen species at 24 hours and blastocyst development after 48 and 72 hours in the control group $(r=1, P<.05)$. However, in embryos that were cultured in HTF-alone, a negative correlation was seen between the levels of reactive oxygen species at 48 hours and 72 hours with hatching of blastocysts $(r=-0.81, P<.005)$. A significant correlation was seen between the levels of reactive oxygen species at 48 hours

Table 3. Apoptosis in Blastocyst After Culture in HTF-alone and HTF-SSS

\begin{tabular}{lcccc}
\hline \multicolumn{1}{c}{ Group } & $\begin{array}{c}\text { Apoptotic } \\
\text { Blastocyst }\end{array}$ & $\begin{array}{c}\text { Apoptosis per } \\
\text { Blastocyst }\end{array}$ & $\begin{array}{c}\text { Apoptosis in } \\
\text { Inner Cell Mass }\end{array}$ & $\begin{array}{c}\text { Apoptosis in } \\
\text { Trophectoderm }\end{array}$ \\
\hline HTF-alone & $65(98)$ & $5.81 \pm 1.11$ & $2.51 \pm 0.75$ & $3.50(1.14)$ \\
HTF-SSS & $61(94)$ & $2.38 \pm 0.68$ & $1.52 \pm 0.41$ & $1.19(0.69)$ \\
$P$ & .30 & $<.001$ & $<.001$ & $<.001$ \\
\hline
\end{tabular}

HTF, human tubal fluid; SSS, serum substitute supplement.

Values are $\mathrm{n}(\%)$ or mean \pm standard deviation.

$P<.05$ was considered significant using generalized estimating equation logistic regression for percentage of apoptotic blastocysts and mixed-model analysis of variance for the remaining variables. 
and the incidence of apoptosis in the HTF-alone group $(r=1, P<.0001)$.

\section{DISCUSSION}

Several major developmental events take place in the embryos during the postfertilization window. ${ }^{3,8}$ These include the first cleavage division, ${ }^{19}$ the switching on of the embryonic genome, ${ }^{30}$ and compaction of the morula. Clearly, any modifications of the culture environment, which could affect any or all of these processes, could have a major effect on the quality of the embryo. Efforts to improve culture media raise the question of whether degree of fragmentation and developmental potential, cell number, and apoptosis bear any correlation with embryos cultured in different media. Although apoptosis takes place as a normal part of embryonic development in vivo, it is more likely to occur during in vitro embryo culture due to suboptimal conditions. ${ }^{12-14}$ Paradoxically, higher levels of apoptosis have been reported even in embryos of excellent morphology, suggesting a possible role in regulation of cell number ${ }^{31}$ and preventing overgrowth of the inner cell mass. ${ }^{32}$ Spontaneous apoptosis occurs frequently in in vitro cultured bovine morulae/blastocysts when cultured under both low oxygen $^{33}$ and high oxygen tension. ${ }^{12}$ Oviductal and uterine environment where early embryos develop naturally are characterized by an oxygen tension approximately one quarter to one third of atmospheric oxygen tensions.

Supraphysiologic oxygen tension can generate the formation of reactive oxygen species during embryo culture. ${ }^{9,34}$ In our present study, the effect of protein supplementation using 10\% serum substitute supplement on reactive oxygen species production and apoptosis in mouse embryos was examined. Our results demonstrate that despite a similar rate of development and embryo quality between the HTF-alone and HTF-SSS culture systems, profound differences existed at the molecular level for apoptosis and amount of reactive oxygen species. Use of $10 \%$ serum substitute supplement in HTF medium may serve as a survival factor and a reactive oxygen species scavenger to suppress cell death in the preimplantation embryos. In our study, we did not detect apoptosis in earlier developmental stages (4-cell to morula). This is similar to the findings of other investigators. ${ }^{35,36}$ Supplementation of the media with protein significantly improved the blastocyst hatching. The hatchability of embryos cultured in HTF-alone could be rescued by returning the embryos to HTF medium supplemented with serum at the early blastocyst stage. ${ }^{37}$ The zona pellucida of mouse oocytes undergoes spontaneous zona hardening when cultured in serum-free medium. ${ }^{38}$ This renders oocytes refractory to sperm pene- tration, fertilization, and development. Addition of serum to culture medium has been shown to inhibit spontaneous zona hardening. ${ }^{39}$ The serum substitute supplement used in our present study as the protein source is a defined mixture of $84 \%$ human serum albumin and 16\% human globulins. These globulins (predominantly $\alpha$ - and $\beta$-globulins) are known to promote mouse embryo development and in particular the blastocyst hatching. ${ }^{40}$ It seems that $\alpha$ - and $\beta$-globulins and/or other unknown growth-promoting factors in the globulin fraction in 10\% serum substitute supplement may stimulate the blastocyst hatching. Similar effects on hatching have been observed by adding growth factors to the culture medium. ${ }^{41}$

The negative correlation between the amount of reactive oxygen species and blastocyst hatching in embryos cultured in HTF-alone observed in our study may perhaps be due to lack of the promoting effect of protein supplementation on blastocyst hatching. Upon differentiation at the early blastocyst stage, trophectoderm secretes zona lysin, which is necessary for zona hatching. ${ }^{42}$ However, continued exposure to increasing levels of reactive oxygen species and the lack of or inadequate antioxidant capacity in the culture environment could affect normal cell function. This results in a decrease in the total lysin production by trophectoderm cells below the threshold level necessary to promote zona thinning. This subsequently inhibits blastocyst hatching. ${ }^{37}$

Excessive production of intracellular reactive oxygen species during embryo culture is generally thought to be detrimental to the embryo development. In our present study we found a slightly higher development rate and total cell number in embryos cultured in HTF-alone compared with HTF-SSS, which might be due to the reactive oxygen species promoting effect on embryo development. Also we found a positive correlation, although not statistically significant, between the amount of reactive oxygen species produced and the embryo development rate. The slightly higher development rate and total cell number observed in embryos cultured in HTF-alone compared with HTF-SSS might be due to the reactive oxygen species promoting effect on embryo development. In the spermatozoa, small amounts of reactive oxygen species are necessary for capacitation, hyperactivation, acrosome reaction, and oocyte fusion. ${ }^{43}$

Presence of antioxidants can exert a beneficial effect on embryo development and influence the incidence of apoptosis. The increase in cell death in blastocyst formation in vitro may be attributed either to the absence of growth or survival factors from the maternal tract or to suboptimal culture conditions. ${ }^{36,44}$ Albumin is the major protein component of serum substitute supplement and participates in the antioxidant defense of embryos. Albu- 
min along with other low molecular weight thiol compounds has been used to supplement oocyte maturation media ${ }^{17,23}$ and embryo culture. ${ }^{16,22}$ They serve as precursors for glutathione formation in the embryo. Glutathione is required for detoxification of lipid peroxides and thiolated proteins and may be involved in removal of $\mathrm{H}_{2} \mathrm{O}_{2}$ in embryos. ${ }^{10}$ Several reports have demonstrated that cell death in blastocysts is most predominant in inner cell mass cells and is occasionally seen in trophectodermal lineage. ${ }^{44}$ The differences in metabolic requirement, cell positioning, and microenvironments are considered to be some of the major causes underlying the differential sensitivity of inner cell mass and trophectoderm cell lines to embryotoxic agents. ${ }^{45}$ Although zona-intact trophectodermal vesicles could hatch at normal rates in the absence of inner cell mass, ${ }^{37}$ a critical number of inner cell mass blastomeres are necessary for normal postimplantation development that may be regulated by apoptosis. ${ }^{44}$ In our study, a higher incidence of apoptosis was often detected in the inner cell mass of embryos cultured in HTF - alone, indicating that our findings may also have implications for protein supplementation in culture media.

Blastocoele fluid induces apoptosis in inner cell mass cells, and $\mathrm{H}_{2} \mathrm{O}_{2}$ is the cytotoxic substance present in the blastocoele fluid. ${ }^{46}$ Supplementing the embryo with albumin as we did in our study tends to increase the intracellular glutathione content, resulting in removal of $\mathrm{H}_{2} \mathrm{O}_{2}$ and lowering the incidence of apoptosis in inner cell mass cells. Addition of several growth factors could further decrease cell death. It is likely that $\alpha$ - and $\beta$-globulins or other unknown growth-promoting factors in the globulin fraction of serum substitute supplement may have a similar effect on the incidence of apoptosis in embryos cultured in vitro.

In conclusion, using a serum substitute supplement in culture medium with a well-defined composition of albu$\mathrm{min}$ and globulins is beneficial for the embryos in reducing the incidence of apoptosis. This may help minimize the adverse effects observed by other investigators using fetal calf serum or serum from other biologic sources. Different sources of serum may have other, undefined components that may negate the beneficial effects. The beneficial effects reported in our study may occur by scavenging embryotoxic component(s) such as excessive amount of reactive oxygen species that result in oxidative stress or by closely mimicking in vivo culture conditions possibly due to the presence of certain growth factors. In addition, protein supplementation improves the hatching rate, and this may help increase both implantation and pregnancy. Our study may have implications for human in vitro fertilization and could serve as a model for further research to optimize in vitro culture system for human embryos.

\section{REFERENCES}

1. Boni R, Tosti E, Roviello S, Dale B. Intercellular communication in vivo and in vitro-produced bovine embryos. Biol Reprod 1999;61:1050-5.

2. Viuff D, Rickords L, Offenberg H, Hyttel P, Avery B, Greve T, et al. A high proportion of bovine blastocysts produced in vitro are mixoploid. Biol Reprod 1999;60: 1273-8.

3. Rizos D, Ward F, Duffy P, Boland MP, Lonergan P. Consequences of bovine oocyte maturation, fertilization or early embryo development in vitro versus in vivo: implications for blastocyst yield and blastocyst quality. Mol Reprod Dev 2002;61:234-48.

4. Sinclair KD, Young LE, Wilmut I, McEvoy TG. In-utero overgrowth in ruminants following embryo culture: lessons from mice and a warning to men. Hum Reprod 2000;15(suppl):68-86.

5. Farin PW, Grosier AE, Farin CE. Influence of in vitro systems on embryo survival and fetal development in cattle. Theriogenology 2001;55:151-70.

6. Fair T, Lonergan P, Dinnyes A, Cottell DC, Hyttel P, Ward FA, et al. Ultrastructure of bovine blastocysts following cryopreservation: effect of method of blastocyst production. Mol Reprod Dev 2001;58:186-95.

7. Khurana NK, Niemann H. Effects of oocyte quality, oxygen tension, embryo density, cumulus cells and energy substrates on cleavage and morula/blastocyst formation of bovine embryos. Theriogenology 2000;54:741-56.

8. Rizos D, Lonergan P, Boland MP, Arroyo-Garcia R, Pintado B, de la Fuente J, et al. Analysis of differential messenger RNA expression between bovine blastocysts produced in different culture systems: implications for blastocyst quality. Biol Reprod 2002;66:589-95.

9. Goto Y, Noda Y, Mori T, Nakano M. Increased generation of reactive oxygen species in embryos cultured in vitro. Free Rad Biol Med 1993;15:69-75.

10. Johnson MH, Nasr-Esfahani MH. Radical solutions and cultural problems: could free oxygen radicals be responsible for the impairment of preimplantation mammalian embryos in vitro? BioEssays 1994;16:31-8.

11. Guerin P, El Mouatassim S, Menezo Y. Oxidative stress and protection against reactive oxygen species in the preimplantation embryo and its surroundings. Hum Reprod Update 2001;7:175-89.

12. Matwee C, Betts DH, King WA. Apoptosis in the early bovine embryo. Zygote 2000;8:57-68.

13. Hardy K, Spanos S, Becker D, Iannelli P, Winston RM, Stark J. From cell death to embryo arrest: mathematical models of human preimplantation embryo development. Proc Natl Acad Sci U S A 2001;98:1655-60. 
14. Hardy K. Apoptosis in the human embryo. Rev Reprod 1999;4:125-34.

15. Gjorret JO, Knijn HM, Dieleman SJ, Avery B, Larsson LI, Maddox-Hyttel P. Chronology of apoptosis in bovine embryos produced in vivo and in vitro. Biol Reprod 2003;69:1193-2000.

16. Caamano JN, Ryoo ZY, Thomas JA, Youngs CR. Betamercaptoethanol enhances blastocyst formation rate of bovine in vitro-matured/in vitro-fertilized embryos. Biol Reprod 1996;55:1179-84.

17. De Matos DG, Furnus CG, Moses DF, Baldassarre H. Effect of cysteamine on glutathione level and developmental capacity of bovine oocyte matured in vitro. Mol Reprod Dev 1995;42:432-6.

18. Wang X, Falcone T, Attaran M, GoldbergJM, Agarwal A, Sharma RK. Vitamin G and vitamin E supplementation reduce oxidative stress-induced embryo toxicity and improve the blastocyst development rate. Fertil Steril 2002;78:1272-7.

19. Lonergan P, Khatir H, Piumi F, Rieger D, Humblot P, Boland MP. Effect of time interval from insemination to first cleavage on the developmental characteristics, sex ratio and pregnancy rate after transfer of bovine embryos. J Reprod Fertil 1999;117:159-67.

20. Iwata H, Akamatsu S, Minami N, Yamada M. Effects of antioxidants on the development of bovine IVM/IVF embryos in various concentrations of glucose. Theriogenology 1998;50:365-75.

21. Fujitani Y, Kasai K, Ohtani S, Nishimura K, Yamada M, Utsumi K. Effect of oxygen concentration and free radicals on in vitro development of in vitro-produced bovine embryos. J Anim Sci 1997;75:483-9.

22. Takahashi M, Nagai T, Hamano S, Kuwayama M, Okamura N, Okano A. Effect of thiol compounds on in vitro development and intracellular glutathione content of bovine embryos. Biol Reprod 1993;49:228-32.

23. De Matos DG, Furnus CC, Moses DF. Glutathione synthesis during in vitro maturation of bovine oocytes: role of cumulus cells. Biol Reprod 1997;57:1420-5.

24. Liu Z, Foote RH. Development of bovine embryos in KSOM with added superoxide dismutase and taurine and with five and twenty percent $\mathrm{O}_{2}$. Biol Reprod 1995;53: 786-90.

25. Iwata H, Akamatsu S, Minami N, Yamada M. Allopurinol, an inhibitor of xanthine oxidase, improves the development of IVM/IVF bovine embryos ( $>4$ cell) in vitro under certain culture conditions. Theriogenology 1999;51: 613-22.

26. Salas-Vidal E, Lomeli H, Castro-Obregon S, Guervo R, Escalante-Alcalde D, Covarrubias L. Reactive oxygen species participate in the control of mouse embryonic cell death. Exp Cell Res 1998;238:136-47.

27. Attaran M, Pasqualotto E, Falcone T, GoldbergJM, Miller
$\mathrm{KF}$, Agarwal A, et al. The effect of follicular fluid reactive oxygen species on the outcome of in vitro fertilization. Int J Fertil Womens Med 2000;45:314-20.

28. Darzynkiewicz Z, Bruno S, Del Bino G, Gorczycza W, Hotz MA, Lassota P, et al. Features of apoptotic cells measured by flow cytometry. Cytometry 1992;13: 795-808.

29. Brison DR, Schultz RM. Increased incidence of apoptosis in transforming growth factor alpha-deficient mouse blastocysts. Biol Reprod 1998;59:136-44.

30. Memili E, First NL. Zygotic and embryonic gene expression in cow: a review of timing and mechanisms of early gene expression as compared with other species. Zygote 2000;8:87-96.

31. Hardy K, Stark J, Winston RM. Maintenance of the inner cell mass in human blastocysts from fragmented embryos. Biol Reprod 2003;68:1165-69.

32. Leese HJ, Donnay I, Thompson JG. Human assisted conception: a cautionary tale. Lessons from domestic animals. Hum Reprod 1998;13:184-202.

33. Byrne AT, Southgate J, Brison DR, Leese HJ. Analysis of apoptosis in the preimplantation bovine embryo using TUNEL. J Reprod Fertil 1999;117:97-105.

34. Nasr-Esfahani MH, Aitken JR, Johnson MH. Hydrogen peroxide levels in mouse oocytes and early cleavage stage embryos developed in vitro or in vivo. Development 1990; 109:501-7.

35. Handyside AH, Hunter S. Cell division and death in the mouse blastocyst before implantation. Rouxs Arch Dev Biol 1986;86:519-26.

36. Xu J, Cheung TM, Chan ST, Ho PC, Yeung WS. Human oviductal cells reduce the incidence of apoptosis in cocultured mouse embryos. Fertil Steril 2000;74:1215-9.

37. Schiewe MG, Hazeleger NL, Sclimenti C, Balmaceda JP. Physiological characterization of blastocyst hatching mechanisms by use of a mouse antihatching model. Fertil Steril 1995;63:288-94.

38. De Felici M, Siracusa G. Spontaneous hardening of zona pellucida of mouse oocytes during in vitro culture. Gamete Res 1982;6:107-13.

39. Dell'Aquila ME, De Felici M, Massari S, Maritato F, Minioa P. Effects of fetuin on zona pellucida hardening and fertilizability of equine oocytes matured in vitro. Biol Reprod 1999;61:533-40.

40. Tanikawa M, Harada T, Ito M, Yoshida S, Iwabe T, Terakawa N. Globulins in protein supplements promote the development of preimplantation embryos. J Assist Reprod Genet 1999;16:555-57.

41. Onohara Y, Harada T, Tanikawa M, Iwabe T, Yoshioka $\mathrm{H}$, Taniguchi $\mathrm{F}$, et al. Autocrine effects of transforming growth factor-alpha on the development of preimplantation mouse embryos. J Assist Reprod Genet 1998;15: 395-402. 
42. Perona RM, Wassarman PM. Mouse blastocysts hatch in vitro by using a trypsin-like proteinase associated with cells of mural trophectoderm. Dev Biol 1986;14:42-52.

43. De Lamirande E, Gagnon C. Human sperm hyperactivation and capacitation as parts of an oxidative process. Free Radic Biol Med 1993;14:157-66.

44. Brison DR, Schultz RM. Apoptosis during mouse blastocyst formation: evidence for a role for survival factors including transforming growth factor alpha. Biol Reprod 1997;56:1088-96.

45. Pampfer S. Apoptosis in rodent peri-implantation embryos: differential susceptibility of inner cell mass and trophectoderm cell lineages-a review. Placenta 2000;21(suppl): S3-10.
46. Parchment RE. The implications of a unified theory of programmed cell death, polyamines, oxyradicals and histogenesis in the embryo. Int J Dev Biol 1993;37:75-83.

Reprints are not available. Address correspondence to: Rakesh K. Sharma, PhD, Center for Advanced Research in Human Reproduction, Infertility, and Sexual Function, Department of Obstetrics-Gynecology and Glickman Urological Institute, 9500 Euclid Avenue, Desk A19.1, Cleveland, Ohio 44195; e-mail: sharmar@ccf.org.

Received Fuly 19, 2004. Received in revised form October 8, 2004. Accepted November 4, 2004. 